\title{
One Step Surfactant Free Synthesis and Characterization of Spherical Gold Nanoparticles
}

\author{
D. Ragupathy ${ }^{1, *}$, E. Muthusankar ${ }^{1,2}$, Narendran Rajagopalan ${ }^{3}$
}

${ }^{1}$ Department of Chemistry, National Institute of Technology Puducherry, Karaikal - 609 609, Puducherry, India.

${ }^{2}$ Department of Electronics and Communication Engineering, National Institute of Technology Puducherry, Karaikal - 609 609, Puducherry, India.

${ }^{3}$ Department of Computer Science and Engineering, National Institute of Technology Puducherry, Karaikal - 609 609, Puducherry, India.

\section{A R T IC LE DETAILS}

\section{Article history:}

Received 03 January 2018

Accepted 15 January 2018

Available online 04 February 2018

\section{Keywords:}

Gold Nanoparticles

Surfactant Free

TEM

\begin{abstract}
A B S T R A C T
We report a novel and simple one step method for the synthesis of spherical gold nanoparticles (SGNPs) by the reduction of gold (III) chloride trihydrate $\left(\mathrm{HAuCl}_{4}\right)$ through a surfactant free single step method. High resolution transmission electron microscopy (HRTEM) was evaluated to depict the morphology of SGNPs. TEM images reveal the homogeneous spherical gold nanoparticles formation. The average diameter of single SGNPs was calculated to be $\sim 2.5-5 \mathrm{~nm}$. In addition, the existence of gold nanoparticles was investigated by X-ray photoelectron spectroscopy (XPS) and UV-visible spectroscopy studies. Also, Surface plasmon resonance peak of SGNPs recorded via UV-visible spectroscopy. We expect the method introduced in our work may be more useful for the large-scale synthesis of gold nanomaterials and for multifunctional applications.
\end{abstract}

\section{Introduction}

In the recent years, the synthesis and application of metal nanoparticles have attracted considerable attention due to their unique physical and chemical properties [1,2]. There is enormous interest in metal nanoparticles, i.e. particles in the approximate size range $1-10 \mathrm{~nm}$ have been evolved. These are used in a variety of applications, the most important of which is catalysis and industrial processes such as petroleum reforming depend on catalytic metal nanoparticles, as do the catalytic converters used to clean up motor vehicle exhausts. Metal nanoparticles are also used in a range of other applications, including the labeling of biological samples and hydrogen storage. One of the challenges in this field is to make the particles as small as possible, with as narrow as possible a range of diameters. Since from the discovery of gold nanoparticles (GNPs) by the citrate reduction of $\mathrm{HAuCl}_{4}$ reported by Turkevitch et al., [3] GNPs paid much attention by the researchers and scientists because of their classic and excellent stability. Also, utilization of metal nanoparticles in electrochromic devices, optical devices, biological sensing, catalysis, etc., [4-7] depends on the size of the nanoparticles.

Gold and other noble metal nanocrystals find importance in different branches of science [8] such as catalysis, catalysts for the growth of nanowires, nano-medicines, nano-electronics, etc. For most of those applications, their size and size distributions are of crest importance. To utilize the size and quantum confinement effects of GNPs and to tailor properties of GNPs, it is very important to prepare the defined size and mono-dispersed GNPs synthesis. Different methodologies were adopted to prepare the GNPs such as, micelle or reverse micelle, laser ablation and wet chemistry $[9,10]$. Nowadays, utilization of GNPs becomes most important in various fields. In addition, the method for preparing GNPs with novel shape, structures and effortless is still limited. On this basis, more and more attentions have been devoted to the synthesis and characterization of well-defined metal nanostructures such as nanowires, nanorods, nanospheres, square, triangles, cubes and branched particles etc.

Recently, one-step synthesis as a very simple procedure of preparing metal nanoparticles and nanowires has been extensively studied [12]. For the reason of large scale practical applications, it is necessary to find some cheap chemical agents to act as precursors. Considering the above grounds, we report a facile and surfactant free a synthesis of SGNPs using

sodium borohydrate as reducing agent to reduce $\mathrm{HAuCl}_{4}$ through a single step method.

\section{Experimental Methods}

\subsection{Materials}

Gold (III) chloride trihydrate $\left(\mathrm{HAuCl}_{4} \cdot 3 \mathrm{H}_{2} \mathrm{O}\right)$, sodium borohydrate $\left(\mathrm{NaBH}_{4}\right)$ and ethanol were purchased from Sigma (Korea). All chemicals were used without further purification. Distilled water was used throughout the experiments.

\subsection{Synthesis of Spherical Gold Nanoparticles (SNGP'S)}

Gold nanoparticles were prepared according to the following procedure: First the aqueous solutions of $\mathrm{HAuCl}_{4} \cdot 3 \mathrm{H}_{2} \mathrm{O}(0.054 \mathrm{mM}, 5 \mathrm{~mL})$ was stirred under vigorous condition. Then $2 \mathrm{~mL}$ of freshly prepared $\mathrm{NaBH}_{4}(0.2 \mathrm{mM})$ aqueous solution was added drop by drop to the above stirred solution and continuously stirring for overnight at room temperature. Gold nanoparticles solution was centrifuged three times at $3500 \mathrm{rpm} / \mathrm{min}$ at room temperature for $30 \mathrm{~min}$ to remove other unwanted impurities as much as possible.

The mechanistic formation of SGNPs exists in two stages: In the first stage, while vigorous stirring $\mathrm{Cl}^{-}$ions are exchanged against $\mathrm{AuCl}_{4}^{-}$ions. In the next stage GNPs are generated by reduction of the confined $\mathrm{AuCl}_{4}$ counter-ions by $\mathrm{NaBH}_{4}$ as shown in Scheme 1.

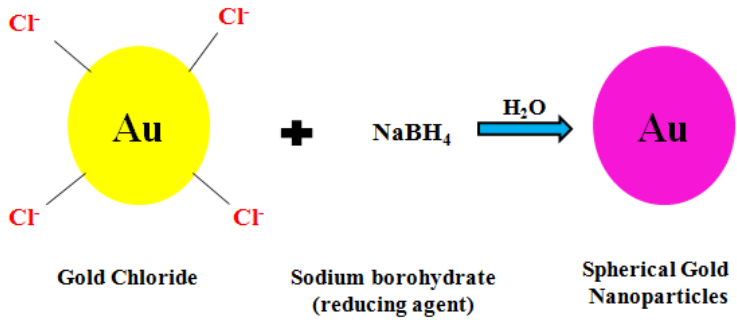

Scheme 1 Preparation of spherical gold nanoparticles (SGNPs)

\subsection{Characterization Techniques}

The morphology and size of the SGNPs were investigated by Hitachi HF2000 (HRTEM) with an accelerating voltage of $200 \mathrm{kV}$. TEM suspension was made by dispersion of SGNPs in ethanol under ultrasonication and 
then placing a drop of dilute suspension on a carbon coated copper grid. UV-visible spectra of the samples were recorded using Schimadzu UVvisible spectrophotometer. X-ray photoelectron spectroscopy (XPS) was used to characterize the SGNPs.

\section{Results and Discussion}

\subsection{Morphological Studies}

Gold nanoparticles have been achieved by the typical reduction method of gold solution at room temperature. Fig. 1a and b shows the typical TEM images of SGNPs from lower to higher magnification. In the Fig. 1a shows the aggregation of uniform sized SGNPs. Fig. 1c displays the single spherical gold nanoparticles with an average diameter of 2.5 to $5 \mathrm{~nm}$. Generally, $5-15 \mathrm{~nm}$ size of metal nanoparticles was exist in the spherical or sphere form reported by Dabbousi et al., [13]. This gold nanoparticle was expected to possess surface plasmon resonance because of the free electrons travel in the metal (d electrons in gold). Fig. 1c reveals the single spherical gold nanoparticle existence. In addition, Fig. 1c inset shows the Fast Fourier Transformation (FFT) of SGNP exhibit three intense rings, suggesting the preferential crystal growth in one direction.

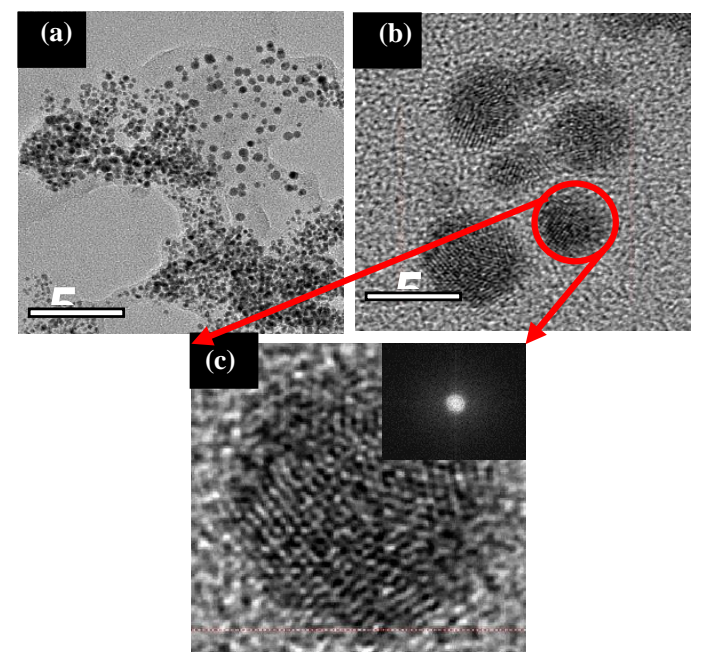

Fig. 1 TEM images $(\mathrm{a}-\mathrm{c})$ of the SGNPs from lower to higher magnification and (c) inset shows the FFT image of single SGNP

\subsection{Elemental Analysis Technique}

$\mathrm{X}$-ray photoelectron spectroscopy (XPS) is a quantitative spectroscopic technique that measures the elemental composition, empirical formula, chemical state and electronic state of the elements that exist within a material. Here, the oxidation state of gold nanoparticles was confirmed by XPS studies. Fig. 2 presents the XPS spectrum of GNPs showing the characteristic $\mathrm{Au} ; 4 \mathrm{f} 7 / 2$ and $4 \mathrm{f} 5 / 2$ doublets. The clear peaks centered at $83.1 \mathrm{eV}$ and $87.3 \mathrm{eV}$ are the existence of SGNP's [14].

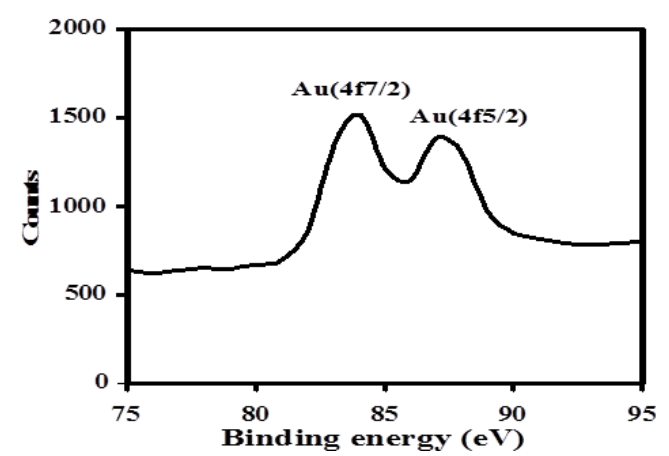

Fig. 2 XPS spectrum of SGNPs showing $A u(4 f 7 / 2)$ and $A u(4 f 5 / 2)$ double peaks

\subsection{UV-Visible Spectroscopy Studies}

UV-visible spectroscopy is one of the most important techniques to ascertain the formation and stability of metal nanoparticles in aqueous solution. Fig. 3 shows the UV-visible spectra of SGNPs in water solution. UV-visible spectrum of SGNPs shows peak at $530 \mathrm{~nm}$ is due to the size, shape and surface plasmon resonance (SPR) effect of the gold nanoparticles [15]. Usually for the spherical nanoparticles, the SPR band maximum generally falls between about 520 and $540 \mathrm{~nm}$ [16].

https://doi.org/10.30799/jnst.094.18040102

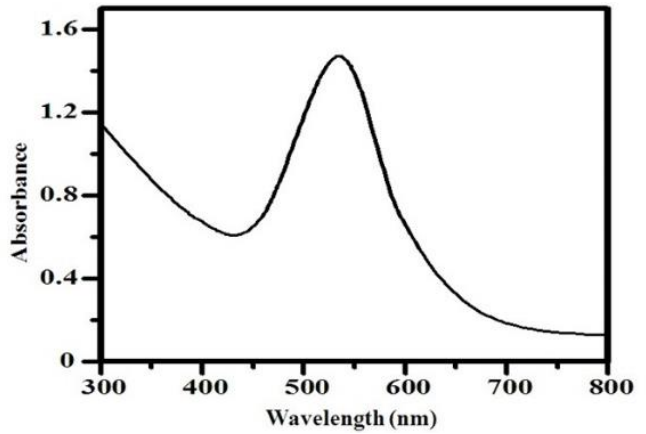

Fig. 3 UV-vis spectrum of SGNPs

\section{Conclusion}

In summary, we report a novel and simple method for the synthesis of spherical gold nanoparticles through a surfactant free single step method. Transmission electron microscopy (TEM) was evaluated to analyze the morphology and formation of SGNPs. The average diameter of single SGNPs was calculated to be $\sim 2.5-5 \mathrm{~nm}$. The SPR property of the SGNPs was analyzed by optical measurement. We anticipated that this method would be more useful for the large-scale synthesis of gold nanomaterials and find applications in electro-catalyst, biosensors etc.

\section{Acknowledgements}

The authors gratefully acknowledge the support from Science and Engineering Research Board - Department of Science Technology grant (SB/FT/CS-117/2014). The authors acknowledge the basic research support from National Institute of Technology, Puducherry.

\section{References}

[1] A.N. Shipway, E. Katz, I. Willner, Nanoparticle arrays on surfaces for electronic, optical, and sensor applications, Chem. Phys. Chem. 1 (2000) 18-52.

[2] M.C. Daniel, A. Didier, Gold nanoparticles: assembly, supramolecular chemistry, quantum-size-related properties, and applications toward biology, catalysis, and nanotechnology, Chem. Rev. 104 (2004) 293-346.

[3] J. Turkevich, P.C. Stevenson, J. Hillier, A study of the nucleation and growth processes in the synthesis of colloidal gold, Disc. Faraday Soc. 11 (1951) 55-75.

[4] H. Jiangtao, T.W. Odom, C.M. Lieber, Chemistry and physics in one dimension: synthesis and properties of nanowires and nanotubes, Acc. Chem. Res. 32 (1999) 435-445.

[5] S.A. Maier, P.G. Kik, H.A. Atwater, S. Meltzer, E. Harel, B.E. Koel, A.A.G. Requicha, Local detection of electromagnetic energy transport below the diffraction limit in metal nanoparticle plasmon waveguides, Nat. Mater. 2 (2003) 229-232.

[6] W.C.W. Chan, S. Nie, Quantum dot bioconjugates for ultrasensitive nonisotopic detection, Sci. 281 (1998) 2016-2018.

[7] J.J. Storhoff, R. Elghanian, R.C. Mucic, C.A. Mirkin, R.L. Letsinger, One-pot colorimetric differentiation of polynucleotides with single base imperfections using gold nanoparticle probes, J. Am. Chem. Soc. 120 (1998) 1959-1964.

[8] H.S. Kimberly, J.J. Schwartz, A.T. Santos, S. Zhang, J.M. Jacobson, Remote electronic control of DNA hybridization through inductive coupling to an attached metal nanocrystal antenna, Nature 415 (2002) 152-155.

[9] C. Petit, P. Lixon, M.P. Pileni, In situ synthesis of silver nanocluster in AOT reverse micelles, J. Phys. Chem. 97 (1993) 12974-12983.

[10] F. Mafune, J. Kohno, Y. Takeda, T. Kondow, Formation and size control of silver nanoparticles by laser ablation in aqueous solution, J. Phys. Chem. B 104 (2000) 9111-9117.

[11] I. Lisiecki, Isabelle, M.P. Pileni, Synthesis of copper metallic clusters using reverse micelles as microreactors, J. Am. Chem. Soc. 115 (1993) 3887-3896.

[12] W. Gang, H. Zhou, Z. Liu, Y. Song, L. Wang, L. Sun, Z. Li, One-step synthesis of silver nanoparticles, nanorods, and nanowires on the surface of DNA network, J. Phys. Chem. B 109 (2005) 8738-8743.

[13] B.O. Dabbousi, J.R. Viejo, F.V. Mikulec, J.R. Heine, H. Mattoussi, R. Ober, et al., (CdSe) ZnS core- shell quantum dots: synthesis and characterization of a size series of highly luminescent nanocrystallites, J. Phys. Chem. B. 101 (1997) 9463-9475.

[14] K.M. Manesh, J.H. Kim, P. Santhosh, A.I. Gopalan, K.P. Lee, H.D. Kang, Fabrication of a gold nanoparticles decorated carbon nanotubes based novel modified electrode for the electrochemical detection of glucose, J. Nanosci. Nanotechnol. 7 (2007) 3365-3372.

[15] J.H. Kim, K.S. Kim, K.M. Manesh, P. Santhosh, A.I. Gopalan, K.P. Lee, Selfassembly directed synthesis of gold nanostructures, Colloid. Surf. A: Physicochem. Eng. Aspect. 313 (2008) 612-616.

[16] T. Shimizu, T. Teranishi, S. Hasegawa, M. Miyake, Size evolution of alkanethiolprotected gold nanoparticles by heat treatment in the solid state, J. Phys. Chem. B 107 (2003) 2719-2724. 